\title{
ON THE ESTIMATION OF UPPER BOUND FOR SOLUTIONS OF PERTURBED DISCRETE LYAPUNOV EQUATIONS
}

DONG-YAN CHEN AND DE-YU WANG

Received 20 February 2006; Revised 4 June 2006; Accepted 12 June 2006

The estimation of the positive definite solutions to perturbed discrete Lyapunov equations is discussed. Several upper bounds of the positive definite solutions are obtained when the perturbation parameters are norm-bounded uncertain. In the derivation of the bounds, one only needs to deal with eigenvalues of matrices and linear matrix inequalities, and thus avoids solving high-order algebraic equations. A numerical example is presented.

Copyright (C) 2006 D.-Y. Chen and D.-Y. Wang. This is an open access article distributed under the Creative Commons Attribution License, which permits unrestricted use, distribution, and reproduction in any medium, provided the original work is properly cited.

\section{Introduction}

Consider the following perturbed discrete Lyapunov equation for the variable matrix $P \in$ $\mathbb{R}^{n \times n}$ :

$$
P=(A+\Delta A)^{T} P(A+\Delta A)+Q
$$

where the matrix $A \in \mathbb{R}^{n \times n}$ is given, $\Delta A \in \mathbb{R}^{n \times n}$ is an uncertain matrix which represents the structure disturbance of $A$, and $Q \in \mathbb{R}^{n \times n}$ is a symmetric positive definite or semidefinite matrix.

Assume that $\Delta A$ satisfies the norm-bounded uncertainty

$$
\triangle A=D F E
$$

where $D$ and $E$ are given constant matrices of appropriate dimensions, and $F$ is an unknown real time-varying matrix with Lebesgue measurable entries satisfying $F^{T} F \leq I$ with $I$ being an identity matrix of appropriate dimension. Furthermore, we assume that $A$ is asymptotically stable.

The discrete Lyapunov equation (1.1) plays an indispensable role in many areas of science and technology, such as system design, signal processing and optimal control, and so forth. Hence, the investigation on its solutions is very important. Recently, there have 
been a lot of results obtained on this aspect and we refer to the survey paper [3] and references therein. The estimation on the solutions of discrete Lyapunov equation is getting more and more accurate. But in practice, perturbed discrete Lyapunov equation is much more involved, since model error or unmodel dynamic state cannot be avoided. So determining the bounds of positive definite or positive semidefinite solutions of perturbed discrete Lyapunov equation possesses more practical values. This problem has been studied in [7], where the solution of a fourth-order algebraic matrix equation is required during the derivation of the bounds, and the numerical aspect has not been discussed. In the present paper, we derive the bounds of solutions to (1.1) through a simple way by straightforwardly applying the properties of matrix eigenvalues and some matrix inequalities. Moreover, the uncertainty considered in this paper is much more general than that in [7].

\section{Main results}

We first fix some notations which will be used throughout the paper: $\mathbb{R}^{n \times n}$ is the set of $n \times n$ real matrices; $\operatorname{tr}(X), \lambda_{i}(X)$, and $\operatorname{det}(X)$ denote, respectively, the trace, $i$ th eigenvalue, and determinant of matrix $X \in \mathbb{R}^{n \times n}$. The eigenvalues are assumed to be arranged in decreasing order, that is,

$$
\left|\lambda_{1}(X)\right| \geq\left|\lambda_{2}(X)\right| \geq \cdots \geq\left|\lambda_{n}(X)\right|
$$

The abbreviation SPD stands for "symmetric positive definite," while SPSD stands for "symmetric positive semidefinite."

Next, we give some preliminary lemmas for the subsequent use.

Lemma 2.1 [5]. Suppose A, D, E are given constant matrices of appropriate dimensions and $F$ is an uncertain matrix satisfying $F^{T} F \leq I$. Let $P$ be an SPD matrix and let $\varepsilon>0$ be a constant. Then, if $P-\varepsilon D D^{T}>0$, it holds that

$$
(A+D F E)^{T} P^{-1}(A+D F E) \leq A^{T}\left(P-\varepsilon D D^{T}\right)^{-1} A+\frac{1}{\varepsilon} E^{T} E .
$$

Lemma 2.2 [1]. For any real symmetric matrices $X$ and $Y$, the following inequalities hold:

$$
\begin{aligned}
& \lambda_{1}(X+Y) \leq \lambda_{1}(X)+\lambda_{1}(Y), \\
& \lambda_{n}(X+Y) \geq \lambda_{n}(X)+\lambda_{n}(Y) .
\end{aligned}
$$

Lemma 2.3 [2]. Matrix $\left[\begin{array}{ll}A & B \\ C & D\end{array}\right]>0(<0)$ if and only if (a) $D>0(<0)$ and $A-B D^{-1} C>$ $0(<0)$ or $(b) A>0(<0)$ and $D-C A^{-1} B>0(<0)$.

Lemma 2.4 [6]. Let $Y, M$, and $N$ be constant matrices of appropriate dimensions and, in particular, let $Y$ be symmetric. For any matrix $F$ satisfying $F^{T} F \leq I$, the inequality

$$
Y+M F N+N^{T} F^{T} M^{T}<0
$$


holds if and only if there is a constant $\varepsilon>0$, such that

$$
\varepsilon^{2} M M^{T}+\varepsilon Y+N^{T} N<0 .
$$

Lemma 2.5. The following statements are equivalent:

(a) there exists a matrix $P_{1}$ such that $P_{1}=P_{1}^{T}>0$ and

$$
A^{T} P_{1} A-P_{1}+Q<0
$$

(b) there exists a symmetric positive semidefinite solution matrix $P_{2}$ to the Lyapunov equation

$$
A^{T} P_{2} A-P_{2}+Q=0
$$

Furthermore, if the above conditions hold, then $P_{2}<P_{1}$.

Proof. The lemma is a straightforward corollary of [7, Theorem 7.2.2].

Now, we are ready to present the main results.

THEOREM 2.6. If there is a constant $\varepsilon>0$ such that

$$
\begin{gathered}
\lambda_{1}\left(A^{T}\left(I-\varepsilon D^{T} D\right)^{-1} A+\frac{1}{\varepsilon} E^{T} E\right)<1, \\
I-\varepsilon D^{T} D>0
\end{gathered}
$$

then the solution of the perturbed discrete Lyapunov equation (1.1) satisfies the following inequality:

$$
P \leq \frac{\lambda_{1}(Q)\left[A^{T}\left(I-\varepsilon D^{T} D\right)^{-1} A+(1 / \mathcal{E}) E^{T} E\right]}{1-\lambda_{1}\left(A^{T}\left(I-\varepsilon D^{T} D\right)^{-1} A+(1 / \varepsilon) E^{T} E\right)} .
$$

Proof. Let $P$ be a solution of the perturbed discrete Lyapunov equation (1.1). Then for all $x \in \mathbb{R}^{n}, x \neq 0$, we have

$$
\begin{aligned}
x^{T} P x & =x^{T}(A+\Delta A)^{T} P(A+\Delta A) x+x^{T} Q x \\
& \leq \lambda_{1}(P) x^{T}(A+\Delta A)^{T}(A+\Delta A) x+x^{T} Q x .
\end{aligned}
$$

By Lemma 2.1, it holds that

$$
(A+\Delta A)^{T}(A+\Delta A) \leq A^{T}\left(I-\varepsilon D D^{T}\right)^{-1} A+\frac{1}{\varepsilon} E^{T} E .
$$


4 Upper bound to perturbed DLE

Then, by combining (2.11) and (2.12), we obtain

$$
P \leq \lambda_{1}(P)\left[A^{T}\left(I-\varepsilon D D^{T}\right)^{-1} A+\frac{1}{\varepsilon} E^{T} E\right]+Q .
$$

Taking the maximum eigenvalue $\lambda_{1}(\cdot)$ on both sides of (2.13), and by using Lemma 2.2, we further get

$$
\lambda_{1}(P) \leq \lambda_{1}(P) \lambda_{1}\left(A^{T}\left(I-\varepsilon D D^{T}\right)^{-1} A+\frac{1}{\varepsilon} E^{T} E\right)+\lambda_{1}(Q),
$$

which together with (2.8) implies

$$
\lambda_{1}(P) \leq \frac{\lambda_{1}(Q)}{1-\lambda_{1}\left(A^{T}\left(I-\varepsilon D^{T} D\right)^{-1} A+(1 / \varepsilon) E^{T} E\right)} .
$$

Now, (2.10) follows directly from (2.13) and (2.15).

Theorem 2.7. For any $\varepsilon>0$, set

$$
\begin{gathered}
a=\frac{b-\sqrt{b^{2}-c}}{2 \varepsilon \lambda_{1}\left(D D^{T}\right)}, \\
b=1-\lambda_{1}\left(A^{T} A\right)+\varepsilon \lambda_{1}\left(D D^{T}\right) \lambda_{1}\left(\frac{1}{\varepsilon} E^{T} E+Q\right), \\
c=4 \varepsilon \lambda_{1}\left(D D^{T}\right) \lambda_{1}\left(\frac{1}{\varepsilon} E^{T} E+Q\right) .
\end{gathered}
$$

If there exists $\varepsilon>0$, such that $P^{-1}-\varepsilon D D^{T}>0$ and $b>0, b^{2} \geq c$, then the solution of (1.1) satisfies the following inequality:

$$
P \leq \frac{a A^{T}}{1-\varepsilon a \lambda_{1}\left(D D^{T}\right)}+\frac{1}{\varepsilon} E^{T} E+Q .
$$

Proof. By Lemma 2.1, it holds that

$$
P \leq A^{T}\left(P-\varepsilon D D^{T}\right)^{-1} A+\frac{1}{\varepsilon} E^{T} E+Q .
$$

Using the properties of matrix eigenvalues, we have

$$
\begin{aligned}
A^{T}\left(P^{-1}-\varepsilon D D^{T}\right)^{-1} A & \leq \lambda_{1}\left(\left(P^{-1}-\varepsilon D D^{T}\right)^{-1}\right) A^{T} A \\
& =\frac{1}{\lambda_{n}\left(P^{-1}-\varepsilon D D^{T}\right)} A^{T} A \\
& \leq \frac{1}{1 / \lambda_{1}(P)-\varepsilon \lambda_{1}\left(D D^{T}\right)} A^{T} A \\
& \leq \frac{\lambda_{1}(P)}{1-\varepsilon \lambda_{1}(P) \lambda_{1}\left(D D^{T}\right)} A^{T} A,
\end{aligned}
$$


which when applied to (2.18) gives

$$
P \leq \frac{\lambda_{1}(P)}{1-\varepsilon \lambda_{1}(P) \lambda_{1}\left(D D^{T}\right)} A^{T} A+\frac{1}{\varepsilon} E^{T} E+Q .
$$

Taking the maximum eigenvalues $\lambda_{1}(\cdot)$ on both sides of $(2.20)$, we obtain

$$
\varepsilon \lambda_{1}\left(D D^{T}\right) \lambda_{1}^{2}(P)+\left[\lambda_{1}\left(A^{T} A\right)-\varepsilon \lambda_{1}\left(D D^{T}\right) \lambda_{1}\left(\frac{1}{\varepsilon} E^{T} E+Q\right)-1\right] \lambda_{1}(P)+\lambda_{1}\left(\frac{1}{\varepsilon} E^{T} E+Q\right) \geq 0,
$$

which then implies

$$
\lambda_{1}(P) \leq \frac{b-\sqrt{b^{2}-c}}{2 \varepsilon \lambda_{1}\left(D D^{T}\right)}=a,
$$

where $a, b, c$ are defined in the statement of the theorem. Finally, from (2.20) and (2.22), we get (2.17). The proof is completed.

THeorem 2.8. If there exist an SPD matrix $\bar{X}$ and a constant $\varepsilon>0$ satisfying the linear matrix inequality (LMI)

$$
\left[\begin{array}{cccc}
-\frac{1}{\varepsilon} I & D^{T} \bar{X} & 0 & 0 \\
\bar{X} D & -\bar{X} & \bar{X} A & 0 \\
0 & A^{T} \bar{X} & -\bar{X}+Q & E^{T} \\
0 & 0 & E & -\varepsilon I
\end{array}\right]<0,
$$

then (1.1) has positive definite solutions $P$ and $P<\bar{X}$.

Proof. Since

$$
\begin{gathered}
{\left[\begin{array}{cccc}
-\frac{1}{\varepsilon} I & D^{T} \bar{X} & 0 & 0 \\
\bar{X} D & \bar{X} & \bar{X} A & 0 \\
0 & A^{T} \bar{X} & -\bar{X}+Q & E^{T} \\
0 & 0 & E & -\varepsilon I
\end{array}\right]} \\
=\left[\begin{array}{cccc}
I & 0 & 0 & 0 \\
0 & \bar{X} & 0 & 0 \\
0 & 0 & I & 0 \\
0 & 0 & 0 & I
\end{array}\right]\left[\begin{array}{cccc}
-\frac{1}{\varepsilon} & D^{T} & 0 & 0 \\
D & -\bar{X}^{-1} & A & 0 \\
0 & A^{T} & -\bar{X}+Q & E^{T} \\
0 & 0 & E & -\varepsilon I
\end{array}\right]\left[\begin{array}{cccc}
I & 0 & 0 & 0 \\
0 & \bar{X} & 0 & 0 \\
0 & 0 & I & 0 \\
0 & 0 & 0 & I
\end{array}\right]
\end{gathered}
$$


6 Upper bound to perturbed DLE

and therefore if (2.23) holds, we have

$$
\left[\begin{array}{cccc}
-\frac{1}{\varepsilon} I & D^{T} & 0 & 0 \\
D & -\bar{X}^{-1} & A & 0 \\
0 & A^{T} & -\bar{X}+Q & E^{T} \\
0 & 0 & E & -\varepsilon I
\end{array}\right]<0
$$

By Lemma 2.3, it holds that

$$
\left[\begin{array}{ccc}
-\bar{X}^{-1}+\varepsilon D D^{T} & A & 0 \\
A^{T} & -\bar{X}+Q & E^{T} \\
0 & E & -\varepsilon I
\end{array}\right]<0
$$

and furthermore

$$
\begin{gathered}
{\left[\begin{array}{cc}
-\bar{X}^{-1}+\varepsilon D D^{T} & A \\
A^{T} & -\bar{X}+Q+\frac{1}{\varepsilon} E^{T} E
\end{array}\right]} \\
\quad=\left[\begin{array}{cc}
-\bar{X}^{-1} & A \\
A^{T} & -\bar{X}+Q
\end{array}\right]+\varepsilon\left[\begin{array}{c}
D \\
0
\end{array}\right]\left[\begin{array}{ll}
D^{T} & 0
\end{array}\right]+\frac{1}{\varepsilon}\left[\begin{array}{c}
0 \\
E^{T}
\end{array}\right]\left[\begin{array}{ll}
0 & E
\end{array}\right]<0 .
\end{gathered}
$$

By using Lemma 2.4, we obtain

$$
\left[\begin{array}{cc}
-\bar{X}^{-1} & A \\
A^{T} & -\bar{X}+Q
\end{array}\right]+\left[\begin{array}{c}
0 \\
E^{T}
\end{array}\right] F^{T}\left[\begin{array}{ll}
D^{T} & 0
\end{array}\right]+\left[\begin{array}{c}
D \\
0
\end{array}\right] F\left[\begin{array}{ll}
0 & E
\end{array}\right]<0,
$$

that is,

$$
\left[\begin{array}{cc}
-\bar{X}^{-1} & A+\Delta A \\
A^{T}+\Delta A^{T} & -\bar{X}+Q
\end{array}\right]<0
$$

Next, by Lemma 2.3, we further obtain

$$
(A+\Delta A)^{T} \bar{X}(A+\Delta A)-\bar{X}+Q<0,
$$

which immediately implies that $\bar{X}$ satisfies the inequality corresponding to (1.1).

Finally, by Lemma 2.5, we know that there exist positive definite solutions $P$ to (1.1) and $P<\bar{X}$. The proof is completed.

Remark 2.9. From the relations between the solution of the perturbed discrete Lyapunov equation and that of an appropriate perturbed discrete Riccati equation (see [4]), we know that the upper bound of the matrix solution in Theorem 2.7 is also an upper bound of the matrix solution to the corresponding perturbed discrete Riccati equation. 
Remark 2.10. The upper bounds for the trace, eigenvalue, and determinant of the solution to (1.1) can also be obtained similarly.

Remark 2.11. Existing results on the bound of solutions to (1.1) are scarce, since it usually heavily depends on the estimations of solutions to some corresponding Riccati equation. But it is always very difficult to handle with the Riccati equation. Sometimes, in practice, we only need an effective estimation of the solutions, hence the results in this paper cannot be directly compared with the above-mentioned existing results. Due to space limitation, we only give one example to illustrate the effectiveness of our results in the section which follows.

\section{Numerical example}

In the perturbed discrete Lyapunov equation (1.1), let

$$
\begin{aligned}
A & =\left[\begin{array}{cc}
0.5 & 0.1 \\
0 & 0.4
\end{array}\right], \quad Q=\left[\begin{array}{cc}
0.223 & 0 \\
0 & 0.1
\end{array}\right], \\
\Delta A & =M F N=\left[\begin{array}{ll}
0.049 & 0.014 \\
0.014 & 0.038
\end{array}\right]\left[\begin{array}{cc}
\sin \beta & 0 \\
0 & \cos \beta
\end{array}\right]\left[\begin{array}{ll}
1 & 0 \\
0 & 1
\end{array}\right] .
\end{aligned}
$$

Taking $\varepsilon=2$ in (2.10) and (2.17), we obtain the solutions, respectively,

$$
\begin{aligned}
& P \leq P_{1}=\left[\begin{array}{ll}
0.4169 & 0.0222 \\
0.0222 & 0.2591
\end{array}\right], \\
& P \leq P_{2}=\left[\begin{array}{ll}
0.5707 & 0.0295 \\
0.0295 & 0.4004
\end{array}\right],
\end{aligned}
$$

and clearly $P_{2} \geq P_{1}$.

\section{Acknowledgments}

The authors wish to thank the anonymous referees for their constructive comments and helpful suggestions, which led to a great improvement of the presentation of the paper. The work of Dong-Yan Chen was supported by the National Natural Science Foundation of China under Grant 10471031.

\section{References}

[1] A. R. Amir-Moéz, Extreme properties of eigenvalues of a hermitian transformation and singular values of the sum and product of linear transformations, Duke Mathematical Journal 23 (1956), 463-476.

[2] R. A. Horn and C. R. Johnson, Matrix Analysis, Cambridge University Press, Cambridge, 1985.

[3] W. H. Kwon, Y. S. Moon, and S. C. Ahn, Bounds in algebraic Riccati and Lyapunov equations: a survey and some new results, International Journal of Control 64 (1996), no. 3, 377-389.

[4] L. Z. Lu, Matrix bounds and simple iterations for positive semidefinite solutions to discrete-time algebraic Riccati equations, Journal of Xiamen University. Natural Science 34 (1995), no. 4, 512516. 


\section{Upper bound to perturbed DLE}

[5] S. O. R. Moheimani and I. R. Petersen, Optimal quadratic guaranteed cost control of a class of uncertaintime-delay systems, IEE Proceedings-Control Theory and Applications 144 (1997), no. $2,183-188$.

[6] L. Xie, Output feedback $H_{\infty}$ control of systems with parameter uncertainty, International Journal of Control 63 (1996), no. 4, 741-750.

[7] J.-H. Xu, R. E. Skelton, and G. Zhu, Upper and lower covariance bounds for perturbed linear systems, IEEE Transactions on Automatic Control 35 (1990), no. 8, 944-948.

Dong-Yan Chen: Applied Science College, Harbin University of Science and Technology,

Harbin 150080, China

E-mail address: dychen@hrbust.edu.cn

De-Yu Wang: Applied Science College, Harbin University of Science and Technology,

Harbin 150080, China

E-mail address: w_deyu@yahoo.com.cn 